PROCEEDINGS OF THE

AMERICAN MATHEMATICAL SOCIETY

Volume 135, Number 5, May 2007, Pages 1551-1555

S 0002-9939(06)08613-8

Article electronically published on November 13, 2006

\title{
EXCEPTIONAL SEQUENCES OF EIGENFUNCTIONS FOR HYPERBOLIC MANIFOLDS
}

\author{
HAROLD DONNELLY
}

(Communicated by Mikhail Shubin)

\begin{abstract}
Examples are given of hyperbolic manifolds in every dimension at least five which support sequences of eigenfunctions for the Laplacian whose $L^{\infty}$-norms grow as a power of the eigenvalue while their $L^{2}$-norms are one.
\end{abstract}

\section{INTRODUCTION}

Let $M$ be a compact Riemannian manifold. The Laplacian $\Delta$ is a second order elliptic operator canonically associated to the Riemannian metric. It thus follows from standard elliptic theory that $L^{2} M$ admits an orthonormal basis consisting of eigenfunctions $\phi$, i.e. $\Delta \phi=-\lambda \phi$, where $\lambda$ denotes the corresponding eigenvalue. There are finitely many eigenvalues, counted to multiplicity, on each bounded interval of the real line.

A sequence $\phi_{i}$ of eigenfunctions with eigenvalues $\lambda_{i} \rightarrow \infty$ is said to be an exceptional sequence if for some $\epsilon>0$, one has $\left\|\phi_{i}\right\|_{\infty} \geq c \lambda_{i}^{\epsilon}\left\|\phi_{i}\right\|_{2}$. The simplest examples of eigenfunctions are the sine and cosine functions on the circle or flat torus, where the supremum of the eigenfunction is bounded independently of $\lambda$. Separation of variables on the round sphere gives exceptional sequences of eigenfunctions which may concentrate near the poles or the equator. Bourgain 2 showed that exceptional sequences are produced by perturbation of the metric on the flat torus. The author [3] generalized his results to manifolds admitting isometric circle actions. Related work of Toth and Zelditch [12] deals with systems which are quantum completely integrable.

The existence or absence of exceptional sequences for hyperbolic manifolds is less well understood. The first examples were found by Rudnick and Sarnak 8], who proved

Theorem 1. There exist compact manifolds of constant negative curvature and dimension three which support exceptional sequences of eigenfunctions, with $\epsilon=$ $1 / 4$.

The purpose of this paper is to develop their method and thereby establish

Theorem 2. For each $n \geq 5$, there exist compact $n$-dimensional manifolds of constant negative curvature supporting exceptional sequences of eigenfunctions, with $\epsilon=(n-4) / 4$.

Received by the editors October 18, 2004 and, in revised form, December 9, 2005.

2000 Mathematics Subject Classification. Primary 58J50, 58J53.

The author was partially supported by NSF Grant 0203070-DMS. 
Sections 2 and 3 are devoted to the proof of Theorem 2. For dimensions two and four, it remains unclear whether or not there exist hyperbolic manifolds which support exceptional sequences of eigenfunctions. Related problems are discussed in Section 4.

\section{Theta FunCtions}

Let $d$ be a positive square-free integer. Suppose that $K=Q(\sqrt{d})$ and $\mathcal{O}$ is the ring of algebraic integers in $K$. The quadratic form $Q\left(x_{1}, x_{2}, \ldots, x_{n+1}\right)=$ $x_{1}^{2}+x_{2}^{2}+\cdots+x_{n}^{2}-\sqrt{d} x_{n+1}^{2}$ is anisotropic because its conjugate, over the rationals, $\tilde{Q}\left(\tilde{x}_{1}, \tilde{x}_{2}, \ldots, \tilde{x}_{n+1}\right)=\tilde{x}_{1}^{2}+\tilde{x}_{2}^{2}+\cdots+\tilde{x}_{n}^{2}+\sqrt{d} \tilde{x}_{n+1}^{2}$ is positive definite. Let the identity components of the subgroups in $G \ell(n+1, R)$ which preserve $Q$, respectively $\tilde{Q}$, be denoted by $G_{R}$, respectively $\tilde{G}_{R}$. If $G_{\mathcal{O}}$ is the subgroup of $G_{R}$ with entries in $\mathcal{O}$, then we embed $G_{\mathcal{O}}$ into $G_{R} \times \tilde{G}_{R}$ by $g \rightarrow(g, \tilde{g})$. According to Borel and HarishChandra 1, Corollary 12.4, p. 531], the quotient spaces $G_{\mathcal{O}} \backslash G_{R}$ and $G_{\mathcal{O}} \backslash G_{R} \times \tilde{G}_{R}$ are compact. If $K$ denotes a maximal compact subgroup in $G_{R}$, then the quotient spaces $G_{\mathcal{O}} \backslash G_{R} / K$ and $G_{\mathcal{O}} \backslash G_{R} \times \tilde{G}_{R} / K \times \tilde{G}_{R}$ have constant negative curvature.

Suppose that $\hat{Q}(x, y)=Q(x)+\tilde{Q}(y)$ is defined for vectors $(x, y) \in R^{n+1} \times R^{n+1}$. Similarly, we set $\hat{G}=G_{R} \times \tilde{G}_{R}$. If $Q_{1}=2 \hat{Q}$, then let $\hat{R}$ be a majorant of $Q_{1}$, so that $\hat{R}$ is a positive definite matrix satisfying the property $\hat{R} Q_{1}^{-1} \hat{R}=Q_{1}$. We may choose $\hat{R}(x, y)=R(x)+2 \hat{Q}(y)$, where $R(x)$ is a majorant of $Q(x)$. The Gaussian

$$
f(x, y)=e^{-\pi \hat{R}(x, y)}
$$

decomposes as $f(x, y)=f_{1}(x) f_{2}(y)$, where

$$
f_{1}(x)=e^{-\pi R(x)} \quad \text { and } \quad f_{2}(y)=e^{-2 \pi \tilde{Q}(y)} .
$$

The hyperbolic plane $H$ consists of complex numbers with a positive imaginary part. The ingredients collected above are used to construct the $\theta$-function on $H \times H \times \hat{G}$ given by

$$
\theta(z, g)=\left(v_{1} v_{2}\right)^{\frac{n+1}{2}} \sum_{x \in \ell \mathcal{O}} e\left(Q(x) u_{1}+\tilde{Q}(\tilde{x}) u_{2}\right) f_{1}\left(\sqrt{v_{1}} g_{1}^{-1} x\right) f_{2}\left(\sqrt{v_{2}} g_{2}^{-1} \tilde{x}\right)
$$

Here $e$ denotes the exponential $e(\beta)=\exp (2 \pi i \beta)$. Moreover, $z=\left(z_{1}, z_{2}\right)$ is a pair of complex numbers $z_{i}=u_{i}+\sqrt{-1} v_{i}, i=1,2$, and $g=\left(g_{1}, g_{2}\right)$ belongs to $\hat{G}=G_{R} \times \tilde{G}_{R}$. The integer $\ell$ is chosen sufficiently large to permit the application of [10] below.

It is easy to verify that the following equations are valid:

$$
\begin{array}{rlrl}
\theta(z, \gamma g) & =\theta(z, g), & & \gamma \in G_{\mathcal{O}}, \\
\theta(z+(\alpha, \tilde{\alpha}), g) & =\theta(z, g), & \alpha \in \mathcal{O}, \\
\theta(z, g k) & =\theta(z, g), & & k \in K \times \tilde{G}_{R} .
\end{array}
$$

Note that we now suppose that $K$ is chosen by requiring that its elements preserve the majorant $R$.

A deeper property follows by specializing the generalized Poisson summation formula of Shintani [10]. By applying his Propositions 1.6 and 1.7, we deduce that

$$
\theta(\gamma z, g)=\chi(\gamma) \theta(z, g), \quad \gamma \in \Gamma .
$$


Here $\chi$ is a character of an appropriate subgroup $\Gamma$ of finite index in $S L(2, \mathcal{O})$. The subgroup $\Gamma$ is defined by congruence conditions on its entries. If $z=\left(z_{1}, z_{2}\right)$, then $\gamma z=\left(\gamma z_{1}, \tilde{\gamma} z_{2}\right)$.

Another application of Shintani's Proposition 1.7 yields

$$
D_{z} \theta(z, g)=L_{g} \theta(z, g) \text {. }
$$

Here $D_{z}$ is a second order elliptic operator whose leading symbol coincides with the symbol of the Laplacian for the product of hyperbolic metrics on $H \times H$. The operator $D_{z}$ is self-adjoint and acts on sections of the unitary line bundle, over $\Gamma \backslash H \times H$, associated to the character $\chi$. Moreover, $L_{g}$ denotes the Casimir operator of $\hat{G}$ which is self-adjoint with respect to the metric on $G_{\mathcal{O}} \backslash \hat{G} / K \times \tilde{G}_{R}$, induced by the Killing form.

\section{THETA LIFTS}

Suppose that $\phi$ is a square integrable function on the compact quotient $G_{\mathcal{O}} \backslash \hat{G}$. We define the $\theta$-lift of $\phi$ by

$$
F(z)=\int_{G_{\mathcal{O}} \backslash \hat{G}} \theta(z, g) \phi(g) d g .
$$

Integration by parts indicates that this transformation maps eigenfunctions of $L_{g}$ to eigenfunctions of $D_{z}$.

We restrict the domain of the $\theta$-lift to the orthocomplement of the constants, i.e. $\phi$ integrates to zero over $G_{\mathcal{O}} \backslash \hat{G}$. The transformation then has range lying in the cusp forms. For the cusp at infinity one computes

$$
\begin{gathered}
\int_{\mathcal{O} \backslash R^{2}} F(u+i v) d u=\int_{\mathcal{O} \backslash R^{2}} \int_{G_{\mathcal{O}} \backslash \hat{G}} \theta(u+i v, g) \phi(g) d g d u=\left(v_{1} v_{2}\right)^{\frac{n+1}{2}}, \\
\sum_{x \in \ell \mathcal{O}} \int_{\mathcal{O} \backslash R^{2}} e\left(Q(x) u_{1}+\tilde{Q}(\tilde{x}) u_{2}\right) d u \int_{G_{\mathcal{O}} \backslash \hat{G}} f_{1}\left(\sqrt{v_{1}} g_{1}^{-1} x\right) f_{2}\left(\sqrt{v_{2}} g_{2}^{-1} \tilde{x}\right) \phi(g) d g .
\end{gathered}
$$

The integral over $u$ vanishes unless $x=0$. If $x=0$, the functions $f_{1}(0)=f_{2}(0)=1$, and $\phi(g)$ integrates to zero. To check the cuspidal condition at the other cusps of $\Gamma$, one pulls back $\theta(z, g)$ to the cusp at infinity, and uses Proposition 1.7 of Shintani [10] to reduce to the previous calculation.

The Fourier coefficients corresponding to non-zero algebraic integers $\alpha \in \theta$ are defined by

$$
a_{\alpha}\left(v_{1}, v_{2}\right)=\int_{\mathcal{O} \backslash R^{2}} F(u+i v) e\left(-\alpha u_{1}\right) e\left(-\tilde{\alpha} u_{2}\right) d u .
$$

Substituting the definitions of $F$ and $\theta$ gives

$$
a_{\alpha}\left(v_{1}, v_{2}\right)=\left(v_{1} v_{2}\right)^{\frac{n+1}{2}} \sum_{Q(x)=\alpha_{G_{\mathcal{O}} \backslash \hat{G}}} \int_{\phi} \phi(g) f_{1}\left(\sqrt{v_{1}} g_{1}^{-1} x\right) f_{2}\left(\sqrt{v_{2}} g_{2}^{-1} \tilde{x}\right) d g .
$$

To simplify this last expression, one proceeds by using the method of Hejhal [ 6 ] or Rudnick-Sarnak 8. The reader is referred to [8, p. 209] for a thorough explanation. 
If $\alpha<0$ and $\phi$ is a $K \times \tilde{G}_{R}$ invariant eigenfunction with eigenvalue $\lambda$, then

$$
a_{\alpha}\left(v_{1}, v_{2}\right)=\tilde{f}(\lambda, v) \sum_{j=1}^{h} \frac{1}{w_{j}} \phi\left(g_{j}\right) .
$$

Here $w_{j}$ are positive integers and $g_{j} \in \hat{G}$ are certain special values. More specifically, the $w_{j}$ are the orders of the stability groups for the action of $G_{\mathcal{O}}$ on the quadric $Q(x)=\alpha$. The assumption $\alpha<0$ guarantees that each $w_{j}$ is finite. The group elements $g_{j}$ map a basepoint to representatives of the orbits of $G_{\mathcal{O}}$ on this quadric. The function $\tilde{f}$ is a transform of $f$ by integrating against a spherical function with eigenvalue $\lambda$.

If $\phi$ lies in the kernel of the $\theta$-lift, then it follows that

$$
\sum_{j=1}^{h} \frac{1}{w_{j}} \phi\left(g_{j}\right)=0 .
$$

However, the kernel of the theta lift is the orthocomplement of the image of its adjoint, which maps cusp forms to functions on $G_{\mathcal{O}} \backslash \hat{G} / K \times \tilde{G}_{R}$.

Since the Hilbert modular varieties have real dimension four, the upper bound of [5] shows that there are at most $O\left(\lambda^{2}\right)$ cusp forms where $D_{z}$ has eigenvalue less that $\lambda$. If $n \geq 5$, the Weyl asymptotics give $\Omega\left(\lambda^{n / 2}\right)$ eigenfunctions of the Laplacian with eigenvalue less than $\lambda$. If $h=1$, then $\phi\left(g_{1}\right)=0$, except for $0\left(\lambda^{2}\right)$ of these eigenfunctions. This implies that $\left|\phi\left(g_{1}\right)\right| \geq c \lambda^{(n-4) / 4}\|\phi\|_{2}$, for some $\phi$ in the image of the adjoint of the $\theta$-lift. For additional details and the generalization to all $h \geq 1$, see [8, p. 211]. The proof of Theorem 2 is complete.

\section{Open PRoblems}

It remains unclear whether or not exceptional sequences of eigenfunctions exist for hyperbolic manifolds of dimensions two or four. The argument of Rudnick and Sarnak relies upon the existence of anisotropic quadratic forms defined over the rationals. Also, one must lift eigenfunctions from finite volume quotients of $H$ to manifolds of higher dimension. So, exceptional sequences of eigenfunctions are only produced in dimension three. Recall that anisotropic rational quadratic forms of type $(n, 1)$ only exist for $n \leq 3$; see [3, p. 75]. Our argument uses real quadratic extensions of the rationals and lifts from finite volume quotients of $H \times H$ to manifolds of higher dimension. So the method only works for hyperbolic manifolds of dimension at least five.

In dimension two, Sarnak 9] has conjectured that exceptional sequences of eigenfunctions do not exist. Namely, let $M$ be a compact Riemann surface with a metric of strictly negative curvature. If $\phi \in C^{\infty}(M)$ satisfies $\Delta \phi=-\lambda \phi$, then Sarnak conjectures that, for all $\epsilon>0$, one has $\|\phi\|_{\infty} \leq c(\epsilon) \lambda^{\epsilon}\|\phi\|_{2}$. This conjecture is closely related to the Lindelöf hypothesis in analytic number theory.

An antithetical conjecture is that for any compact Riemannian manifold, other than the circle, one may perturb the metric to obtain exceptional sequences of eigenfunctions. Bourgain [2] showed that such perturbation is always possible for the standard flat torus. His example is a prototype for the more general results of 4] concerning manifolds with isometric circle actions. In another direction, Bourgain's example is a special case of the quantum completely integrable systems 
treated by Toth and Zelditch [12. It seems that new techniques are needed to treat perturbations of metrics not having special symmetries.

\section{REFERENCES}

[1] Borel, A. and Harish-Chandra, Arithmetic subgroups of algebraic groups, Annals of Mathematics, 75 (1962), 485-535. MR0147566 (26:5081)

[2] Bourgain, J., Eigenfunction bounds for compact manifolds with integrable geodesic flows, IHES preprint, 1993.

[3] Cassels, J., Rational quadratic forms, Academic Press, N.Y., 1968. MR0522835 (80m:10019)

[4] Donnelly, H., Bounds for eigenfunctions of the Laplacian on compact Riemannian manifolds, Journal of Functional Analysis, 187 (2001), 247-261. MR.1867351(2002k:58060)

[5] Donnelly, H., On the cuspidal spectrum of locally symmetric spaces of finite volume, Journal of Differential Geometry, 17 (1982), 239-253. MR0664496 (83m:58079)

[6] Hejhal, D., A classical approach to a well-known spectral correspondence on quaternion groups, Lecture Notes in Math., vol. 1135, Springer, Berlin, Heidelberg, N.Y., 1985. MR0803353 (87h:11045)

[7] Hirzebruch, F., Hilbert modular surfaces, L'Enseignment Math., 19 (1973), 183-281. MR0393045 (52:13856)

[8] Rudnick, Z. and Sarnak, P., The behavior of eigenstates of arithmetic hyperbolic manifolds, Communications in Math. Physics, 161 (1994), 195-213. MR1266075 (95m:11052)

[9] Sarnak, P., Arithmetic quantum chaos, Israeli Mathematics Conference Proceedings, 8, BarIlan University, 1995, 183-236. MR.1321639 (96d:11059)

[10] Shintani, T., On the construction of holomorphic cusp forms of half integral weight, Nagoya Math. Journal, 58 (1975), 83-126. MR0389772 (52:10603)

[11] Siegel, C., Lectures on advanced analytic number theory, Tata Institute, Bombay, 1961. MR0262150 (41:6760)

[12] Toth, J. and Zelditch, S., $L^{p}$ norms of eigenfunctions in the completely integrable case, Ann. Inst. Henri Poincare, 4 (2003), 343-368. MR1985776 (2004g:58043)

Department of Mathematics, Purdue University, West Lafayette, Indiana 47907

E-mail address: hgd@math.purdue.edu 